\title{
Characterization of micrometric and localised residual stresses on amorphous materials using dispersion of surface acoustic waves
}

\author{
DUQUENNOY Marc*, OUAFTOUH Mohammadi, DEBOUCQ Julien, \\ LEFEBVRE Jean-Etienne, JENOT Frederic and OURAK Mohamed
}

IEMN-DOAE (UMR CNRS 8520), Université de Valenciennes, 59313 Valenciennes, France

*marc.duquennoy@univ-valenciennes.fr

\begin{abstract}
Keywords: Surface acoustic wave, IDT sensor, Residual Stress, Characterization.
\end{abstract}
\begin{abstract}
For amorphous materials such as glass, the fragility of the material can be limited using surface reinforcement by chemical tempering. The principle of chemical tempering consists in forming a localised compression layer on the surface by immersing the glass in a solution of molten potassium nitrate. In this study, dispersion of surface ultrasonic waves caused by the presence of residual surface stresses was studied. The thickness and the level of the stressed surface zones were estimated using an inverse method.
\end{abstract}

\section{Introduction}

The study and control of residual mechanical stress are of increasing importance in numerous fields such as microelectronics and materials with property gradients with a view to meeting new functional requirements. The effects of these stresses can be harmful in certain applications (breakdown phenomenon) and sometimes beneficial like for example to improve the transport properties in silicon or to mechanically reinforce glass [1-2]. In this study, the influence of the localised field of stress, of which the depth is in the order of tens of micrometres, on the propagation of surface waves were studied theoretically and experimentally on chemically tempered amorphous solids. Based on the theory of acoustoelasticity, this study shows the influence of such a field of stress on the propagation of surface waves and in particular highlights dispersion phenomena. But, in order to characterize this dispersion, it was necessary to generate and detect surface waves over a large frequency range. In this study, we developed and used SAW-IDT MEMS. This original solution is based on the development of an interdigital transducer to generate quasi-monochromatic surface waves and to obtain a rapid and accurate estimation of the phase velocity, key information in the characterization of localised stress. Moreover, HF surface waves could be generated over a broad frequency range up to $60 \mathrm{MHz}$ using SAW-IDT MEMS [3].

\section{Theoretical approach}

Acoustoelasticity. Acoustoelasticity, i.e. the dependence of the acoustic wave velocity on the stress in an elastic material, has been studied for more than 40 years [4,5]. Acoustoelasticity is based on a continuum theory of small disturbances (ultrasonic waves) superimposed on an elastically stressed medium formulated by Cauchy in 1929. The general theory of finite deformation was presented by Murnaghan [4] and later in a treatise by Truesdell and Toupin [6]. A modern theory of acoustoelasticity has been presented introducing third-order elastic constants in the constitutive equation [5]. This formalism requires the deformation energy to be developed up to 3rd order, making the theoretical developments very cumbersome. Different developments [7] have all shown that it is possible to introduce the notion of effective elastic constants (EEC), $C_{I J K L}^{a}$ with: $C_{I J K L}^{a}=C_{I J K L}+\delta C_{I J K L}$ where $C_{I J K L}$ corresponds to the second order elastic constants and $\delta C_{I J K L}$ to the disturbance linked to the presence of an applied or residual stress. Through the introduction of EEC, it is possible to use a classic formalism (second order) in the case of stressed materials. A 
stressed material can be considered, via the EEC, as a stress free material presenting second order elastic constants different from the second order elastic constants of an unstressed material [8].

Modelling of structure with localised residual stress. In numerous cases, fields of stress appear during the processing of materials, with sudden variations in temperature often being the cause of residual stress. However, chemical and mechanical causes also exist. In the context of amorphous materials such as glass, the fragility of the material can be limited using surface reinforcement by chemical or thermal tempering. The principle of chemical tempering of glass consists in replacing the sodium ions by more "voluminous" potassium ions. This chemical reaction occurs by diffusion by immersing the glass in a solution of molten potassium nitrate. A rectangular stress profile, also called "U" profile, is thus obtained (Fig.1). An important characteristic of this tempering is the introduction of a localised field of stress on the surface [9].

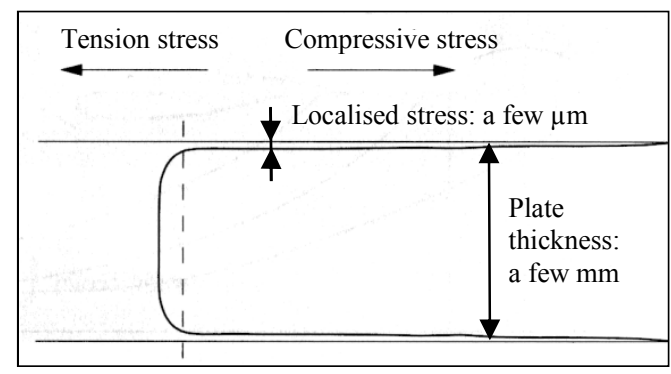

Figure 1. Depth-wise stress profile of chemically tempered glass

This localised stress is a compressive stress which can be in the order of several hundred megapascals and only affects a surface layer of which the thickness is in the order of a few tens of micrometres. Given the "U" (Fig. 1) profile of the field of stress, this chemically stressed structure can be considered as a structure composed of three uniformly stressed parts: firstly, two localised zones (either side of the sheet) of micrometric thickness and under high compressive stress, and secondly, a central zone under low tensile stress. Consequently, given the description of stressed media and effective elastic constants, the chemically stressed glass sheets can be considered as structures made of a layer (one each side) with intrinsic properties different to those of the central substrate. The influence of a localised field of residual stress on the propagation of surface waves on three chemical immersions of 3,12 and 24 hours was thus studied. Considering the surface wave frequencies chosen $(5-60 \mathrm{MHz})$, the chemically stressed sheets of glass were considered here as "layer on semi-infinite substrate"-type structures.

SAW dispersion phenomena. When a SAW (Surface Acoustic Wave) propagates on the surface of a material its energy is concentrated within a thickness of about one wavelength beneath the surface. When this wave propagates in a layer on substrate structure, the surface wave becomes dispersive [10]. The phase and group velocities of this wave partly depend on the characteristics of the layer. In this study, the theoretical dispersion curves were obtained using a program based on a polynomial method allowing the specific acoustic modes of the structure to be determined [11]. In order to calculate the phase velocities of the surface modes, the layer (compressive stress zone) and the substrate (tensile stress zone) were defined by their density and EEC in order to take into account the "perturbation" due to the presence of a residual stress profile. No contraction of indices was performed with the effective elastic constants, thus avoiding splitting that can occur using the contracted notations [8]. The stress field here is however biaxial (plane stress) and isotropic in the plane.

The unordered structure of glass confers it total isotropy and high homogeneity on a macroscopic scale. At ultrasound scale, glass appears homogenous and isotropic. The second- and third-order constants for the glass used in this study were: $C_{11}=84.4 \mathrm{GPa}, \mathrm{C}_{12}=24.8 \mathrm{GPa}, \mathrm{C}_{112}=-80.5 \mathrm{GPa}, \mathrm{C}_{155}=-$ 
27.5GPa and $\mathrm{C}_{456}=-16.5 \mathrm{GPa}$. From the relation between the third-order elastic constants, the following values can be deduced: $\mathrm{C}_{111}=-190.4 \mathrm{GPa}, \mathrm{C}_{123}=-91.3 \mathrm{GPa}, \mathrm{C}_{144}=5.4 \mathrm{GPa}$. The density of the glass was $2508 \mathrm{~kg} \cdot \mathrm{m}^{-3}$ [12]. For the 3 sheets having undergone chemical tempering of 3, 16 and 24 hours, named respectively CT3, CT16 and CT24 (Chemical Tempering, CT), the localised stresses were respectively $-682,-634$ and $-535 \mathrm{MPa}$ and the thicknesses of the compressive fields of stress were respectively $13.4,25.8$ and $34.8 \mu \mathrm{m}$. The theoretical dispersion curves were obtained over a range of frequencies up to $300 \mathrm{MHz}$ and are reported in Fig. 2.

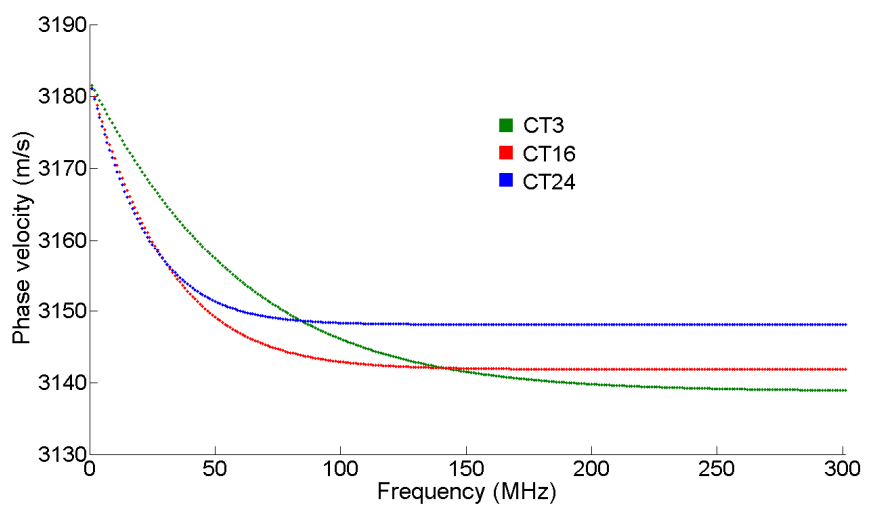

Figure 2. Phase velocities of the first Rayleigh mode propagating on three chemically tempered glass sheets in the frequency range $0-300 \mathrm{MHz}$

It is very interesting to analyse these curves as the influence of the level of localised stress and its depth on the phase velocity of the surface wave can be observed. The phase velocity of the surface wave systematically decreases in the presence of a localised field of stress. The stressed surface zone is called "loading" because it loads the substrate [10]. In this case, the velocity of the surface wave compared with the velocity of the surface wave which would propagate on an unstressed substrate is reduced by the presence of the localised compressive stress. This phenomenon is consistent with the fact that the surface waves are slowed in the presence of a compressive stress [12].

Given that the longer the duration of immersion the greater the thickness of the stressed layer the more the stress decreases, it thus becomes possible to interpret the evolution of the first Rayleigh mode phase velocity as the frequency increases. Indeed, at low frequency, the wavelength of the surface wave is very big in comparison with the thickness of the stressed glass and the wave phase velocity essentially depends on the characteristics of the substrate. Secondly, it is the thickness of the stressed layer which will have the greatest influence on the wave velocity: the greatest decrease in velocity was observed with sheet CT24 immersed for 24 hours (with the thickest stressed layer); this is revealed for the range of frequencies $0-25 \mathrm{MHz}$. At very high frequency (above approximately $150 \mathrm{MHz}$ ), the wavelength is in the order of the depths of the compressive stressed surface zones, which means that the sheet of glass with the highest surface stress will slow the Rayleigh wave the most. The lowest phase velocities can be observed on the dispersion curves for sheet CT3 which has the highest surface stress.

SAW are thus dispersive on a stressed structure at micrometric scale and over a frequency range between 5 and $60 \mathrm{MHz}$ and their phase velocities may decrease by a few tens of meters per second. So, if it is possible to carry out measurements over this wide frequency range, it is possible to accurately characterize the depth of the zone of compressive stress, which is why we have chosen to use SAW to highlight this phenomenon on these structures. 


\section{Measurements}

The main challenge was firstly to develop sensors to generate high frequency SAW over a wide frequency range and secondly to develop a signal processing method to obtain sufficiently accurate estimates of SAW propagation velocities. To meet these challenges, we proposed narrowband SAW-IDT MEMS which offer the possibility of generating quasi-monochromatic surface waves covering a wide range of frequencies $(5-60 \mathrm{MHz})$. It is thus possible to implement techniques for estimating phase velocities as with non-dispersive materials allowing rapid and accurate estimation of SAW phase velocities [3]. The IDT is placed flat and maintained on the sample with an acoustic couplant; the transducer surface, on which the fingers are deposited, is in contact with the sample. For SAW detection, a heterodyne interferometer was used. Good detection levels with good signal to noise ratios have been obtained thanks to the quality of the coupling (Fig. 3).

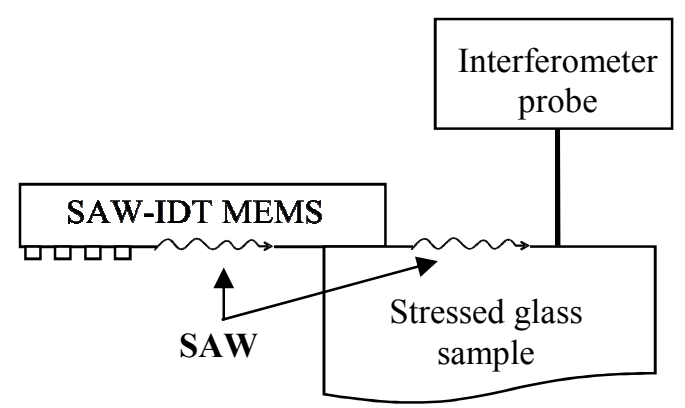

Figure 3. Sketch of generation of surface acoustic waves with SAW-IDT MEMS sensors and detection with interferometer in stressed sample

As an example, Fig. 4 presents the phase velocity measurements obtained for sheet CT3. For the 13 frequencies tested corresponding to the MEMS eigenfrequencies, the means of the phase velocities are represented by dots and the dispersion of these velocities ( \pm standard deviation) by error bars.

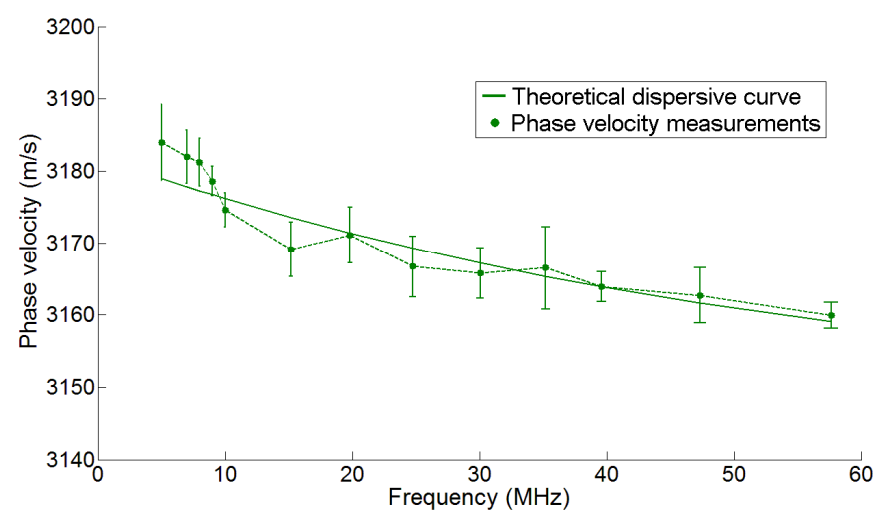

Figure 4. Phase velocities on sheet CT3 and dispersion curve after inversion

An inverse method was developed in order to obtain the thickness of the stressed layer for each of the 3 immersions. A least squares minimization was carried out in order to obtain theoretical propagation velocities as close as possible to the experimental values. The minimization method is based on the dichotomy. It consists in an iterative method of bracketing the desired minimum. Starting from an initial bounded interval which is guaranteed to contain a minimum, the scanning of the interval with different small constant steps allows the width of the bounded interval to be progressively reduced until the desired convergence threshold is reached, i.e. until the width of the 
bounded interval is sufficiently small with a desired tolerance. To solve this inverse problem, 36 velocities were measured between 5 and $60 \mathrm{MHz}$. The theoretical values of the phase velocities were calculated from the stress values and initial thicknesses, the elastic constants and the density for each of the MEMS eigenfrequencies over the entire range of working frequencies. The processing algorithm was then sought to maximize the value of the coefficient of determination, $R^{2}$ [3]. A large interval $(1 \mu \mathrm{m}$ to $101 \mu \mathrm{m})$ for the thickness was chosen to ensure an extremum of $R^{2}$ was obtained [13].

The results obtained for the 3 sheets CT3, CT16 and CT2 4 were 17, 25 and $34 \mu \mathrm{m}$ respectively for the depth and were $-496,-464$ and $-408 \mathrm{MPa}$ respectively for the stress level [14]. The inversion results obtained for the depths of the stressed layers confirm the variation in the depth as a function of the duration of chemical tempering. In addition, these values are in perfect agreement with those predicted by the tempering process implemented. The deviations in the thicknesses of stressed areas are very low and the deviation observed with sheet CT3, although relatively low, could be improved by increasing the range of frequencies measured to over $60 \mathrm{MHz}$. The inversion results relating to the stress values in the surface zones are totally coherent with those of the method of reinforcement by chemical tempering. Indeed, for chemical tempering, the immersion time influences the stress and the depth of the stressed layer. The stress is highest in the sheet for which the tempering was the shortest, which is the case here. However, these values are systematically approximately $25 \%$ lower than the initial values. We think that a progressive relaxation of the stress must have occurred thus leading to this deviation. Nevertheless, these values remain very satisfactory, in the sense that they are consistent with values of compressive stress characteristically obtained with chemical tempering.

\section{Conclusion}

In conclusion, in this study we have shown that the behaviour of a structure with localised residual stress in relation to surface waves is analogous to that of a layer on substrate-type structure. The propagation of surface waves is thus addressed in the context of a classical formalism (second order) with effective elastic constants linked to the state of stress in the layer and the substrate. We have also shown that the depth and stress values of the stressed surface zone can be estimated with good accuracy by resolving the inverse problem.

\section{Acknowledgments}

We would like to thank Nord-Pas-de-Calais and the European Union (FEDER funds) for supporting our research through the CISIT and Interreg IV PRISTIMAT and PRISTIFLEX programs. We would also like to thank Pr. D. Lochegnies and Dr. R. Langlais for providing the glass samples.

\section{References}

[1] P. Fornara, A. Poncet, Modeling of local reduction in TiSi/sub2 and CoSi/sub2 growth near spacers in MOS technologies: influence of mechanical stress and main diffusing species, Int. Electron Devices Meeting Proceedings, San-Francisco, USA 8-11 December (1996) 73-76.

[2] O.S. Narayanaswamy, Stress and structural relaxation in tempering glass, Journal of the American Ceramic Society, 61 (1978) 146-152.

[3] J. Deboucq, M. Duquennoy, M. Ouaftouh, F. Jenot, J. Carlier, M. Ourak, Development of interdigital transducer sensors for non-destructive characterization of thin films using high frequency Rayleigh waves, Review of Scientific Instruments, 82, 064905 (2011) 1-7. 
[4] F.D. Murnaghan, Finite deformation of an elastic solid, Wiley \& Sons edit., Inc. New York (1951) 1-140..

[5] D.S. Hughes, J.L. Kelly, Second-order elastic deformation of solids, Phys. Review, 92, 5 (1953) 1145-1149.

[6] C. Truesdell, R. Toupin, The classical field theories, in Handbuch der Physik, S. Flügge, Ed. Vol.III/1, Springer-Verlag, Berlin and New York (1960) 1-632.

[7] S.H.B. Bosher, D.J. Dunston, Effective elastic constants in nonlinear elasticity, Journal of Applied Physics, 97, 103505 (2005) 1-7.

[8] M. Duquennoy, M. Ouaftouh, D. Devos, F. Jenot, M. Ourak, Effective elastic constants in acoustoelasticity, Applied Physics Letters, 92, 24, 244105 (2008) 1-3.

[9] S. Karlsson, B. Jonson, The technology of chemical glass strengthening - a review, Glass technology-European Journal of Glass Science and Technology Part A, 51 (2010) 41-54.

[10]G.W. Farnell and E.L. Adler, Elastic wave propagation in thin layers, Physical Acoustics edited by W.P. Mason and R.N. Thurston, Academic Press, New York, Vol. 9 (1972) 35-127.

[11]J.E. Lefebvre, V. Zhang, J. Gazalet, T. Gryba, Conceptual advantages and limitations of the Laguerre polynomial approach to analyze surface acoustic waves in semi-infinite substrates and multilayered structures, Journal of Applied Physics 83 (1998) 28-34.

[12]M. Duquennoy, D. Devos, M. Ouaftouh, D. Lochegnies, E. Romero, Ultrasonic evaluation of residual stresses in flat glass tempering : comparing experimental investigation and numerical modelling, Journal of the Acoustical Society of America, 119, 6 (2006) 3773-3781.

[13]M. Duquennoy, M. Ouaftouh, J. Deboucq, J.E. Lefebvre, F. Jenot, M. Ourak, Influence of a superficial field of residual stress on the propagation of surface waves - Applied to the estimation of the depth of the superficial stressed zone, Appl. Phys. Lett., 101, 23 (2012) 1-3.

[14]M. Duquennoy, M. Ouaftouh, J. Deboucq, J.E. Lefebvre, F. Jenot, M. Ourak, Characterization of micrometric and superficial residual stresses using high frequency surface acoustic waves generated by interdigital transducers, J. Acoust. Soc. Am., 134, 6 (2013) 4360-4371. 